\title{
A useful gene cassette for conditional knock-down of essential genes by targeted promoter replacement in Mycobacteria
}

Pauline Texier ${ }^{1}$, Michèle Coddeville ${ }^{1}$, Patricia Bordes ${ }^{1}$ \& Pierre Genevaux ${ }^{*, 1}$

${ }^{1}$ Laboratoire de Microbiologie et de Génétique Moléculaires, Centre de Biologie Intégrative (CBI), Université de Toulouse, CNRS, UPS, 31062 Toulouse Cedex 09, France

BioTechniques 65: 159-162 (September 2018) 10.2144/btn-2018-0074

Keywords: ClpP • Mycobacterium smegmatis $\bullet$ promoter replacement $\bullet$ recombineering $\bullet$ SecA1 • tetracycline promoter

A direct method to study essential genes is to construct conditional knock-down mutants by replacement of their native promoter by an inducible one. In Mycobacteria, replacement of an essential gene promoter with an anhydrotetracycline inducible one was successfully used but required a multi-step approach. In this work, we describe a gene cassette for the engineering of a conditional knock-down mutant, which allows the onestep targeted replacement of mycobacterial promoters by an anhydrotetracycline-inducible promoter. The functionality of this cassette was successfully tested by engineering conditional c/pP and SecA1 mutants of Mycobacterium smegmatis.

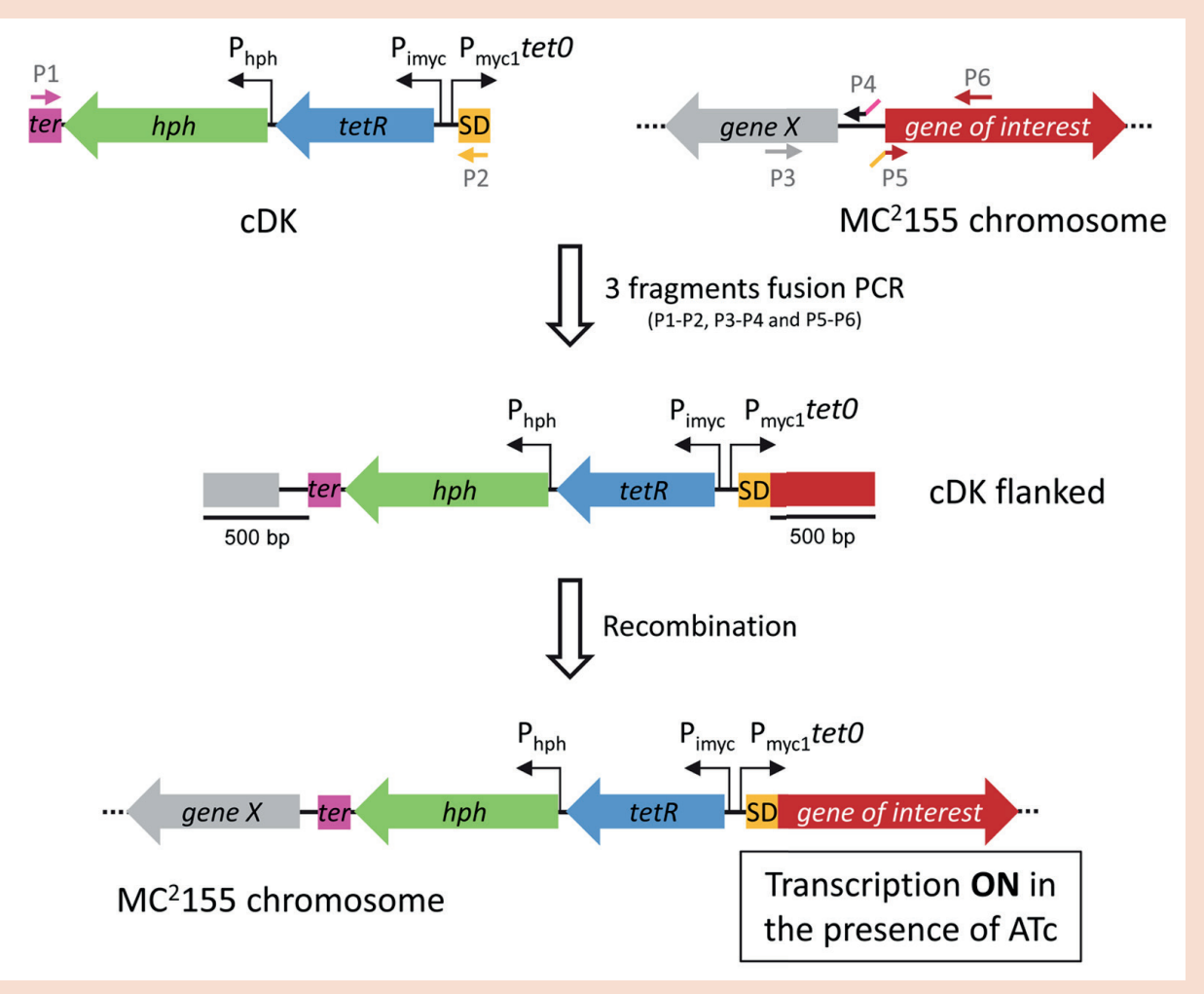

METHOD SUMMARY

The cKD gene cassette allows the one-step replacement of selected promoters in Mycobacteria, including those of essential genes, using the recombineering method. This hygromycin-resistant cassette contains a mycobacterial-adapted TetON promoter system and the Shine-Dalgarno sequence of the dnaK gene, allowing the anhydrotetracycline-dependent expression of a gene of interest. In this work, the CKD cassette was used to replace promoters of both Mycobacterium smegmatis clpP and $\sec A 1$ essential genes. 
Various strategies were developed to study essential genes in Mycobacteria, including conditional expression of antisense RNA [1], CRISPRi-mediated transcriptional repression [2], insertion of a second copy of the essential gene under the control of a inducible promoter followed by deletion of the native copy [3], or replacement of the essential gene native promoter with an inducible one, such as the anhydrotetracycline (ATc)-inducible promoter $\mathrm{P}_{\text {myc1 }}$ tetO locked by the TetR10 repressor. This last method was achieved by recombination of a DNA fragment from either suicide plasmids [4] or temperaturesensitive mycobacteriophages [5]. In this study, we developed and used a new gene cassette, named conditional knock-down (cKD), which allows direct replacement of native mycobacterial promoters by an ATc inducible one, using homologous recombination of linear DNA fragment [6].

The cassette encodes a hygromycinresistance gene and a TetON system composed of the TetR10 repressor and the $\mathrm{P}_{\text {myct }}$ tetO promoter (Figure 1A). This promoter contains 2 tetO surrounding the -35 sequence allowing efficient repression by TetR10 in absence of ATc $[7,8]$. This repression is relieved by ATc that binds to TetR10. Notably, the TetR10 repressor used here, namely $\mathrm{B}_{\text {syn1-207, }}$, was previously adapted for mycobacterial codon usage to allow a more efficient expression and regulation of the $\mathrm{P}_{\text {myct }}$ tetO promoter [9]. Since the Shine-Dalgarno (SD) sequences are absent (leaderless mRNA) or difficult to determine in the GC-rich genome of Mycobacteria [10], we have included a part of $5^{\prime}$-UTR region of the dnaK gene of $M$. tuberculosis, containing the SD and the six nucleotides present between the SD and the initiation codon, at the $3^{\prime}$ end of the cassette (Figure 1A). The cassette can be recombined directly upstream of the start codon of the gene of interest, using the recombineering method [6].

The 2678-nucleotide long cKD cassette was synthetized and cloned as an EcoRV fragment in pUC57 plasmid vector (Genscript, NJ, USA). The sequence is accessible at GenBank, accession number MG674207. The designed cassette was next used for promoter replacement of the M. smegmatis clpP1/P2 operon encoding the AAA+ proteolytic subunit ClpP1/P2 [11]. We expected clpP1 and clpP2 to be essential [11]. The cassette was amplified as

\section{(A)}

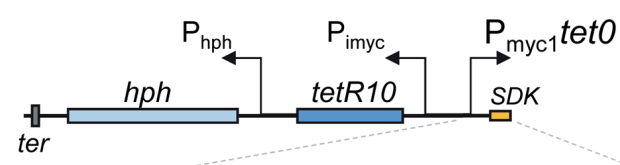

TCCCTATCAGTGATAGAGTIGTCCTCCCTATCAGTGATAGATAGGCTCTGGGAGTACCCGTGTGTACGACCAGCACGGCATACATCATTTCGACG tetO $\quad-35 \quad$ tetO CCGAGAGATTCGCCGCCCGAAATGAGCACGATCCGCATGCACCTCAGGAGGATTCACCATG $S D K$

(B)
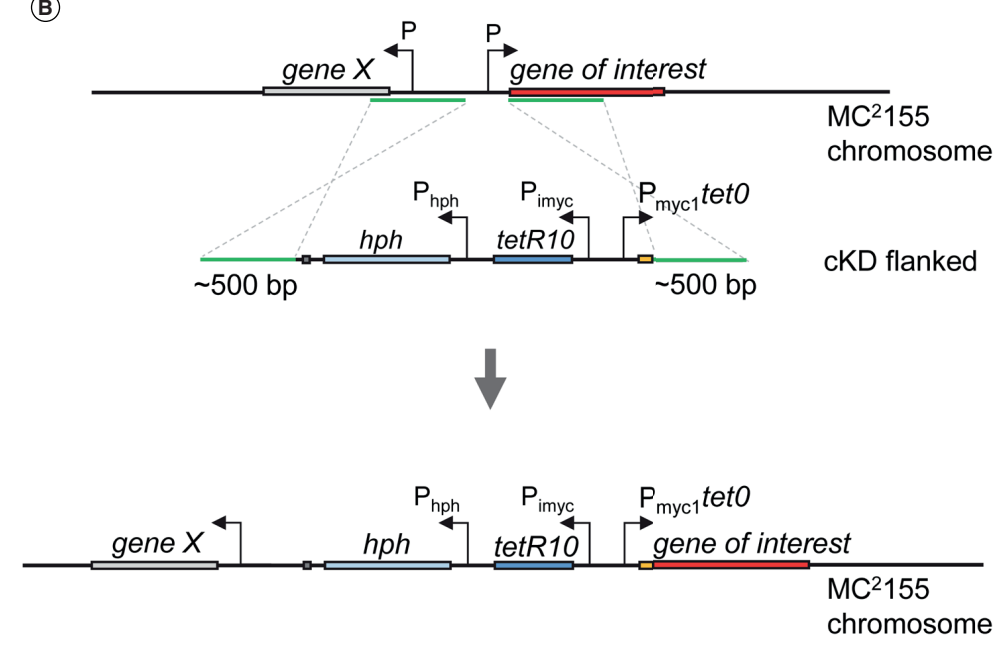

a linear DNA fragment by a two-step fusion PCR in order to add 500-bp flanking regions of homology with the chromosomic region to recombine at each end (Figure 1B). To do so, three fragments corresponding to (i) 500 bp of the upstream genomic region (for clpP1P2:F1_PclpP1P2_For: 5'-caacgcgatccacggcctcg-3' and F1_PclpP1P2_Rev:5'ctgggcctttcgttttattacgggggcgcgttcgc-3), (ii) the cKD cassette (for clpP1P2:F3 PclpP1P2_For:5'-gcgaacgcgcccccgtaata aaacgaaaggcccag-3' and F3_PclpP1P2 Rev:5'-gtgccacgcatgtcagtcacggtgaatcctc ctgaggtg-3') and (iii) the first 500 bp of the gene of interest (for clpP1P2:F2_PclpP1P2 For:5'-cacctcaggaggattcaccgtgactgacatg cgtggcac-3' and F2_PclpP1P2_Rev:5'gtccgaatcggcctcgatgc-3') were amplified by $\mathrm{PCR}$ and fused in a second $\mathrm{PCR}$ reaction, using F1_PclpP1P2_For and F2_PclpP1P2 Rev primers.

The resulting linear DNA was electroporated into M. smegmatis $\mathrm{MC}^{2} 155$ strain containing plasmid pJV53K (kanR), which expresses the gp60 and gp61 recombinases ensuring an elevated level of homologous recombination of the cassette DNA into the chromosome [6]. M. smegmatis hygromycin-resistant clones were selected, the position of the insert into the genome was PCR verified using external primers and correct clones were subjected to successive cycles of growth in liquid $7 \mathrm{H} 9$ medium without kanamycin in order to cure the plasmid pJV53K, as described in [12].

The resulting mutant $\mathrm{MC}^{2} 155$ Ptet-c/pP1/ $P 2$ was grown at $37^{\circ} \mathrm{C}$ in $7 \mathrm{H} 9$ medium supplemented with $0.05 \%$ Tween 20 , $50 \mu \mathrm{g} / \mathrm{mL}$ hygromycin and $20 \mathrm{ng} / \mathrm{mL}$ of ATc inducer. At mid-log phase, the culture

Figure 1. Conditional knock-down mutants by promoter replacement using the CKD cassette. (A) The cKD cassette $(2,7 \mathrm{~KB})$ encodes the TetR10 repressor (dark blue) under the control of the $P_{\text {imyc }}$ promoter, which regulates the $\mathrm{P}$ tetO promoter The upstream UTR region of the dnaK gene covering the Shine-Dalgarno and the six downstream nucleotides (SDK; orange) was placed at the $3^{\prime}$ end of the cassette allowing its insertion just before the start codon of the gene of interest (red [Figure 1B]). This cassette also encodes the hygromycin resistance gene ( $h p h$; light blue) followed by a transcriptional terminator (ter; dark grey) in order to limit polarity on downstream genes. (B) Using fusion $\mathrm{PCR}$, the cKD cassette is flanked by two regions of homology of about 500 nucleotides long (in green) corresponding to the genomic region to recombine. The resulting linear DNA substrate is transformed into the M. smegmatis $\mathrm{MC}^{2} 155$ containing the recombineering plasmid pJV53K, which promotes the subsequent recombination of the flanked cDK into M. smegmatis chromosome. 
(A)

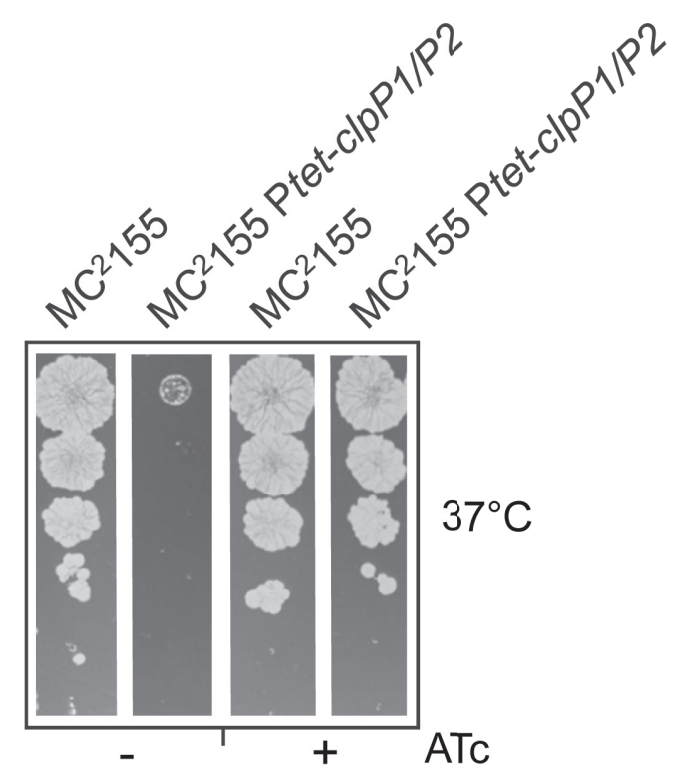

(B)

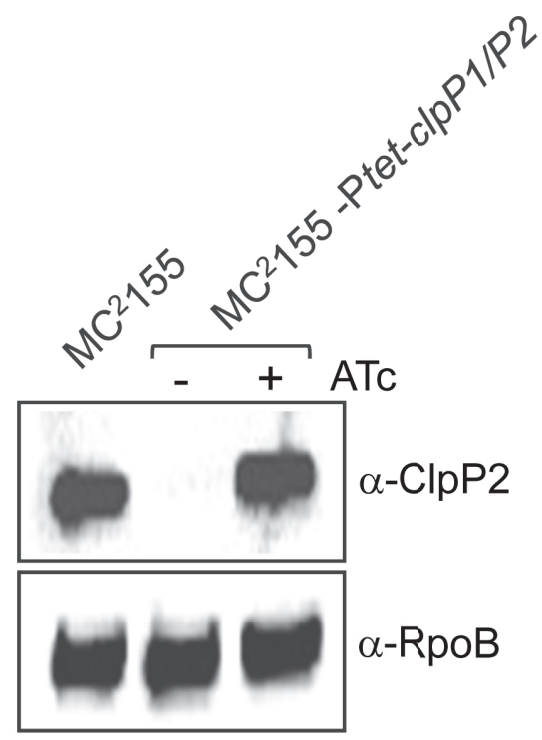

Figure 2. M. smegmatis MC ${ }^{2} 155$ Ptet-clpP1/P2 conditional knock-down mutant. (A) Both $M C^{2} 155$ and $M C^{2} 155$ Ptet-c/pP1/P2 were grown at $37^{\circ} \mathrm{C}$ to mid-log phase, serial diluted and spotted on $7 \mathrm{H} 10$ plates containing hygromycin with (+) or without (-) ATc as indicated. Plates were incubated 3 days at $37^{\circ} \mathrm{C}$. (B) Western blot analysis of ClpP depletion. MC2155 Ptet-clpP1/P2 was grown for 3 days in the presence of $20 \mathrm{ng} / \mathrm{mL}$ ATc inducer, washed three times, diluted in fresh medium with (+) or without (-) inducer and grown to mid-log phase. Whole cell extracts were prepared and the presence of endogenous ClpP2 or RpoB (the RNA polymerase $\beta$ subunit was used as loading control) was visualized by western blot using anti-ClpP2 (a kind gift of David Dougan; La Trobe University, Australia) and anti-RpoB (Neoclone W0023) antibodies. Whole cell extract of $\mathrm{MC}^{2} 155$ wild type (MC $\left.{ }^{2} 155\right)$ was used as a control. ATc: Anhydrotetracycline.

was serially diluted and spotted on agar plates with $7 \mathrm{H} 10$ growth medium containing hygromycin with or without ATc (Figure 2A). After 3 days at $37^{\circ} \mathrm{C}$ we could only detect a few colonies growing at dilution $10^{-1}$, likely extragenous suppressors, when MC $^{2} 155$
Ptet-clpP1/P2 was plated without inducer, indicating that repression of $c / p P 1 / P 2$ in the absence of inducer leads to a severe growth inhibition. In sharp contrast, $\mathrm{MC}^{2} 155$ Ptet-clpP1/P2 growth in the presence of inducer was comparable to that of $\mathrm{MC}^{2} 155$
You care about

\section{ergonomics}

\section{We care about you}

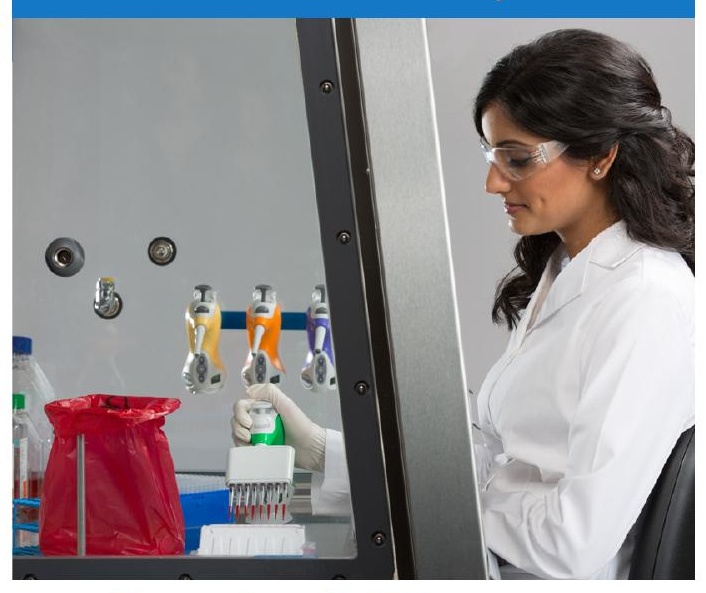

Ovation ${ }^{\circledR}$ Pipettes

\section{Patented ergonomic design \\ made to be better for you}

to make your labwork easier

\section{Recent reviews*}

"Ergonomically made so that elbow is able to rest on benchtop while pipetting."

"I like how well they conform to my hand to reduce wrist and hand strain."

"Easy to use and very accurate. I highly recommend this product for all labs who value preciseness."

\section{More Ovation benefits:}

- Secure tip acquisition with audible "click-on" confirmation so no pounding required

- No-thumb-strain, spring-loaded, easy tip ejection

- Stands on bench even with tip attached to maintain sterility.

- Color coded by volume for easy identification on benchtop

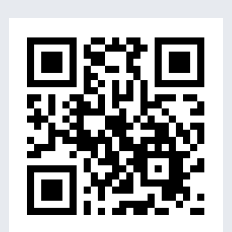

For more ergonomic info and a FREE 2-WEEK TRIAL

"They made the 2-week trial so easy!"

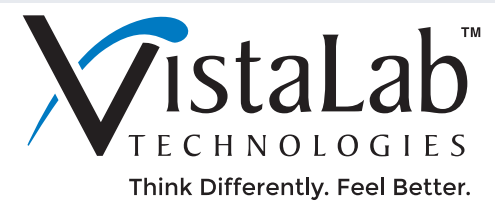

vistalab.com/ovation • +1 914-244-6226 2 Geneva Road, Brewster NY 10509 
wild type (Figure 2A). We next investigated ClpP2 expression in the conditional mutant by western blot analysis. As shown in Figure 2B, ClpP2 was not detected after $24 \mathrm{~h}$ of depletion in the absence of inducer, indicating that the gene was efficiently repressed. By contrast, ClpP2 level in the mutant was comparable to that of the wild-type strain in the presence of inducer (Figure 2B). Note that in addition to clpP1/2, this cassette was successfully used to replace the promoter of the $M$. smegmatis secA1, an essential gene encoding the Sec translocation motor (Supplementary Figure 1). In agreement with the efficiency of the recombineering procedure [6], all of the recombinant strains tested for both c/pP1/2 and secA1 (ten in total) had the insertion at the correct position in the chromosome. Taken together, these results indicate that the newly developed cKD cassette is an efficient tool which allows the one-step engineering of $M$. smegmatis conditional knock-down mutants.

\section{Author contributions}

PT and MC performed experiments. MC and PB performed editing of the manuscript. PG acquired funding. PT and PG contributed to the writing of the manuscript. All the authors contributed to conception and design, analysis and interpretation of data.

\section{Financial \& competing interests disclosure}

This work was supported by FRM-FDT20160435132 fellowships to PT and SNF CRSII3_160703 to PG The authors have no other relevant affiliations or financial involvement with any organization or entity with a financial interest in or financial conflict with the subject matter or materials discussed in the manuscript apart from those disclosed. No writing assistance was utilized in the production of this manuscript.

\section{Open access}

This work is licensed under the Creative Commons Attribution 4.0 License. To view a copy of this license, visit http://creativecommons.org/licenses/by/4.0/

\section{Supplementary data}

To view the supplementary data that accompany this paper please visit the journal website at: https://www.futurescience.com/doi/suppl/10.2144/btn-20180074

\section{References}

Papers of special note have been highlighted as: •• of considerable interest

1. Barik S, Sureka K, Mukherjee P, Basu J, Kundu M. RseA, the SigE specific anti-sigma factor of Mycobacterium tuberculosis, is inactivated by phosphorylation-dependent ClpC1P2 proteolysis. Mol. Microbiol. 75, 592-606 (2010).

2. Rock JM, Hopkins FF, Chavez A et al. Programmable transcriptional repression in mycobacteria using an orthogonal CRISPR interference platform. Nat. Microbiol. 2, 16274 (2017).

3. Schnappinger D, O'Brien KM, Ehrt S Construction of conditional knockdown mutants in mycobacteria. Methods Mol. Biol. 1285, $151-$ 175 (2015).

4. Guo XV, Monteleone M, Klotzsche M et al. Silencing Mycobacterium smegmatis by using tetracycline repressors. J. Bacteriol. 189, 46144623 (2007).

5. Gandotra S, Schnappinger D, Monteleone M, Hillen W, Ehrt S. In vivo gene silencing identifies the Mycobacterium tuberculosis proteasome as essential for the bacteria to persist in mice. Nature Med. 13, 1515-1520 (2007).

6. van Kessel JC, Hatfull GF. Recombineering in Mycobacterium tuberculosis. Nat. Methods 4, 147-152 (2007). -. Describes the recombineering method, a mycobacterial allelic exchange system relying on the expression of mycobacteriophage-encoded recombination proteins.

7. Ehrt S, Guo XV, Hickey CM et al. Controlling gene expression in mycobacteria with anhydrotetracycline and Tet repressor. Nucleic Acids Res. 33, e21 (2005). -• Describes the adaptation of an E. coli tet promoter system for mycobacterial use, allowing an efficient anhydrotetracycline-dependent regulation of the fts $Z$ essential gene.

8. Kaps I, Ehrt S, Seeber D et al. Energy transfer between fluorescent proteins using a co-expression system in Mycobacterium smegmatis. Gene 278, 115-124 (2001).

9. Klotzsche M, Ehrt S, Schnappinger D. Improved tetracycline repressors for gene silencing in mycobacteria. Nucleic Acids Res. 37, 1778-1788 (2009). -. $\quad$ Authors show that codon usage modification of the tetR gene significantly improves TetR repression in Mycobacteria.

10. Cortes T, Schubert OT, Rose G et al. Genomewide mapping of transcriptional start sites defines an extensive leaderless transcriptome in Mycobacterium tuberculosis. Cell Reports 5 , 1121-1131 (2013)

11. Raju RM, Unnikrishnan M, Rubin DH et al. Mycobacterium tuberculosis ClpP1 and ClpP2 function together in protein degradation and are required for viability in vitro and during infection. PLoS Path. 8, e1002511 (2012).

12. Lee MH, Pascopella L, Jacobs WR, Hatfull GF. Site-specific integration of mycobacteriophage L5: integration-proficient vectors for Mycobacterium smegmatis, Mycobacterium tuberculosis, and bacille Calmette-Guerin. Proc. Natl Acad. Sci. USA 88, 3111-3115 (1991).

First draft submitted: 22 May 2018; Accepted for publication: 19 June 2018

Address correspondence to: Pierre Genevaux Laboratoire de Microbiologie et de Génétique Moléculaires, Centre de Biologie Intégrative (CBI), Université de Toulouse, CNRS, UPS, 31062 Toulouse Cedex 09, France; E-mail: genevaux@ibcg.biotoul.fr

To purchase reprints of this article contact: s.cavana@future-science.com 